\title{
CUTANEOUS LUPUS MIMICKING SECONDARY SYPHILIS IN A PATIENT WITH POSITIVE ANTICARDIOLIPIN ANTIBODY - A CASE REPORT
}

Cauê José Pedroso Pinheiro da Silva (Universidade Estadual do Oeste do Paraná, Cascavel, PR, Brasil), Julia Deitos (Universidade Estadual do Oeste do Paraná, Cascavel, PR, Brasil), Andressa Caroline Kuzma (Universidade Estadual do Oeste do Paraná, Cascavel, PR, Brasil), Luisa Manfredin Vila (Universidade Estadual do Oeste do Paraná, Cascavel, PR, Brasil), Jackson Danrlei Balbinot (Universidade Estadual do Oeste do Paraná, Cascavel, PR, Brasil), Lucas Thiesen Pientka (Universidade Estadual do Oeste do Paraná, Cascavel, PR, Brasil), Ana Paula Adame (Universidade Estadual do Oeste do Paraná, Cascavel, PR, Brasil), Marcio Augusto Nogueira (Universidade Estadual do Oeste do Paraná, Cascavel, PR, Brasil)

\section{BACKGROUND}

Systemic lupus erythematosus (SLE) is a chronic autoimmune disease, which etiology is not yet well understood. SLE manifests itself in various systems, such as the skin, joints, central nervous system and kidneys. Syphilis is a disease caused by Treponema pallidum infection, and it is mostly transmitted via direct contact with a syphilitic sore on the skin or mucous membranes. Both diseases, albeit different in their etiopathogenesis, can affect many systems and have similar clinical manifestations. The dermatologic findings of secondary syphilis are variable, and may mimic cutaneous lupus, which can be differentiated by biopsy of the lesion. The symptoms of malar rash and photosensitivity occur in $30-40 \%$ of SLE patients, while dermatological manifestations occur in $88-100 \%$ of cases of secondary syphilis. Nontreponemal tests to diagnosis syphilis have a high number of false-positive results, specially in patients with SLE, antiphospholipid syndrome or other diseases of the connective tissue.

\section{CASE REPORT}

A 27-year-old man presented with a 10-day history of asthenia, hyporexia, somnolence, headaches, dizziness and target-shape erythematous lesions with central paleness on the face, trunk and back, without local pain or other accompanying symptoms. Laboratory investigations revealed a VDRL titer of 1:32, and a negative FTA-Abs. Biopsy of one of the lesions was negative for syphilitic roseola. After discarding the diagnosis of secondary syphilis, the investigation for SLE was initiated. Further laboratory tests showed a positive ANA result (1:640, fine-speckled), and a reagent IgM/IgG anticardiolipin antibody, besides positive results for other autoantibodies in SLE: anti-Sm and anti-dsDNA (1:640). Based on these findings, treatment for SLE was initiated.

\section{CONCLUSION}

Both syphilis and SLE are referred to as "great imitators", and physicians may consider them differential diagnosis for almost any clinical malady. Due to the similar manifestations of those disorders, biopsy of the lesions and laboratory tests are required to arrive at a definitive diagnosis. Our patient had a positive anticardiolipin antibody and dermatologic findings similar to secondary syphilis, which posed a confounding factor in the diagnosis of SLE. 